\title{
Effects of changes in word length on proactive interference in short-term memory
}

\author{
BARBARA LACHAR ${ }^{2}$ and JUDITH \\ GOGGIN, University of Michigan, Ann \\ Arbor, Mich. 48104
}

The effects of changes in word length on proactive interference (PI) were assessed in an STM situation. Four trials with words of the same length or three trials of one length and the fourth with words of the other length were given. Length of the retention interval on Trial 4 was varied. Evidence for $P I$ was found after Trial 1. Recall remained depressed when word length did not change, but retention on Trial 4 improved when word length changed. Variation in retention interval had no effect. The results indicate that word length is a stimulus feature that Ss encode and that a change in this feature reduces interference.

The finding of Peterson \& Peterson (1959), that retention in short-term memory (STM) is a decelerating function of the length of retention interval, stimulated a number of studies designed to extend interference theory to account for losses over short periods of time. Keppel \& Underwood (1962) demonstrated that recall in STM is subject to proactive interference (PI), being a joint function of the number of previously presented items and the length of the retention interval. In an effort to show that the amount of interference depended on item similarity, Wickens, Born, \& Allen (1963) investigated the variable of material class. Holding retention interval constant, they presented a series of consonant trigrams followed by a shift to a series of three-digit numbers, or vice versa. Results indicated a decrease in retention (PI) only when previous items were of the same class as the test items. Immediately upon switching to a new class of material, recall returned to a level equivalent to that found on the first presentation.

Wickens (1968) has since used this situation as a tool to determine the nature of the S's encoding of the stimuli. This procedure is based on the following assumptions: (a) when $S$ is presented a stimulus item, he categorizes or en codes it in a variety of different ways; and (b) the level of recall of an item depends on the similarity between its encoding and the encoding of items that have preceded it. If all the items are similarly categorized, there will be interference and recall will be low; but if the prior items are encoded differently from the to-be-remembered unit, interference will be reduced and recall will be high. Thus, one can discover if an $\mathrm{S}$ has encoded stimulus items on the basis of a particular category by determining whether or not there is a reduction in interference when that category is changed. Positive evidence has been found (Wickens, 1968) for the Ss' encoding of connotative meaning, sense impression, method of presentation, and several other stimulus dimensions.

The present study was designed to determine whether formal stimulus characteristics are also salient dimensions; the particular dimension chosen was word length. The design, similar to that of Hofer (1965), was composed of four trials, with the items on the last trial either similar to or different from the items on Trials 1 to 3 . Length of the retention interval during this critical fourth trial was varied. It was predicted that on "similar" trials, where interference is expected, PI will increase as the retention interval increases. METHOD

The Ss were 184 paid volunteers attending the University of Michigan. Data from $40 \mathrm{Ss}$ were discarded because of a failure to perceive one or more of the items presented. These Ss were not clustered in any particular set of groups. The remaining 144 Ss were equally divided among 12 conditions. Running order was determined by block randomization and, within counterbalancing restrictions, lists and sequence of triads were also randomized.

The learning materials consisted of 24 two-syllable words equally divided into two lengths, 4 letters (short) and 10 letters (long), and matched in frequency of occurrence (Thorndike \& Lorge, 1944). As a result of this restriction, the long words were also compound words. Formal similarity was minimized with no duplication of initial letters, and semantic similarity was low. The words were arranged into eight homogeneous triads (three-word groupings of one length). These items were then used to construct four lists conforming to the following conditions: (a) four long triads, (b) four short triads, (c) three long triads and the fourth triad of short words, and (d) three short triads and the fourth triad of long words. Counterbalancing procedures were used to incorporate four list orders within each condition, with each triad appearing equally often in each position.

Each $S$ first practiced reading numbers for $60 \mathrm{sec}$ in order that he might become accustomed to the pace of the drum. Triads were presented for $1.5 \mathrm{sec}$, during which time Ss pronounced the words aloud. The Ss were instructed to count backward by threes from a three-digit number that appeared immediately after the presentation of each triad. The numbers had been chosen from a table of random numbers, and the same number appeared in each trial across all conditions. Counting continued until three asterisks appeared, signaling spoken recall. The retention interval for Trials 1 to 3 was always $8 \mathrm{sec}$, while the retention interval during the fourth trial varied and was 6,12 , or $18 \mathrm{sec}$. A total of $10 \mathrm{sec}$ was allowed for spoken recall, and there was no intertrial interval. The Ss were asked to recall complete triads, all words in correct sequence, if possible. The four arrangements of word length combine with the three retention intervals to make up 12 experimental conditions

RESULTS

The mean numbers of items recalled on each test, irrespective of whether they occurred in the correct position, are presented in Table 1. A Freeman-Tukey transformation on the scores preceded the analyses. All conditions show PI by the second trial, indicated by the sharp drop in recall. The long-long and short-short conditions show a further decrease in recall from Trial 2 to Trial 3. An analysis of variance on the first three trials showed that the differences between tests were significant $[F(2,284)=86.97, p<.01]$. In addition, short words were recalled better than long words $[F(1,142)=9.97$, $\mathrm{p}<.01]$.

Recall on the fourth trial returns to approximately its original level in the switch conditions, while continuing to decrease in those conditions receiving items of the same length as the preceding trials. An analysis of variance was performed on the total number of words recalled on Trial 4 . The difference between the switch and no-switch conditions was highly reliable $[F(1,132)=108.78, \quad \mathrm{p}<.01]$. This indicates a gross improvement in retention after a switch in presentation to a dissimilar item, regardiess of the length of the items. Only in the short-long and short-short conditions was there a decrease in the mean level of recall as retention interval increased, but neither the overall differences in recall as a function of retention interval nor the anticipated Length Change by Retention Interval interaction approached significance (Fs $<1)$.

Further evidence that PI dissipates or does not occur with a change in the length of the item comes from an examination of the errors made during Trial 4 under various experimental conditions. Only one intraexperimental intrusion appeared in all the switch conditions, while 22 such intrusions occurred when item length did not change. These error frequencies 
Table 1

Mean Numbers of Words Recalled

\begin{tabular}{|c|c|c|c|c|c|c|}
\hline \multirow[b]{3}{*}{ Condition } & \multicolumn{6}{|c|}{ Trials } \\
\hline & \multirow[t]{2}{*}{1} & \multirow[t]{2}{*}{2} & \multirow[t]{2}{*}{3} & \multicolumn{3}{|c|}{4} \\
\hline & & & & $6 \mathrm{Sec}$ & $12 \mathrm{Sec}$ & $18 \mathrm{Sec}$ \\
\hline Long-Short & 2.69 & 1.47 & 1.47 & 2.75 & 2.67 & 2.92 \\
\hline Short-Long & 2.78 & 1.86 & 1.86 & 2.67 & 2.25 & 2.17 \\
\hline Long-Long & 2.53 & 1.81 & 1.17 & 1.08 & .92 & 1.17 \\
\hline Short-Short & 2.78 & 1.78 & 1.50 & 1.33 & 1.33 & 1.17 \\
\hline
\end{tabular}

represent $3 \%$ and $17 \%$ of the total opportunities for error for the switch and no-switch conditions, respectively. DISCUSSION

In this experiment, a switch to different word length on the fourth trial considerably facilitated recall in comparison to groups that received continuous presentations of items of one length. Long words were slightly more difficult to recall than short words even though the lists were matched according to frequency. The fact that long words were also compound may have made them more susceptible to both intra- and extraexperimental interference, although this conclusion was not indicated by the data on overt intrusions.

This study failed to replicate the interaction of similar presentation and length of retention interval found by Hofer (1965). This discrepancy may be due to differences in learning materials, however, since Hofer used word trigrams followed by letter trigrams, and recall was still quite high on Trial 4. In the present study, retention was low on the fourth trial; PI appeared to have already reached an asymptote by $6 \mathrm{sec}$ and remained constant across all retention intervals.

These results reinforce the conclusion reached by Wickens (1968) that Ss can and do respond to many different aspects of the stimulus, both its meaning as well as its formal characteristics. Further research would be required to determine whether $\mathrm{Ss}$ in this study were discriminating between word lengths or between simple vs compound words. Nevertheless, the reduction in interference caused by distinguishably different presentations suggests that even more subtle changes, e.g., varying word endings, may be successful in disrupting the development of PI across trials.

\section{REFERENCES}

HOFER, R. Intertrial proactive inhibition in short-term memory. Psychological Reports, $1965,17,755-760$.

KEPPEL, G., \& UNDERWOOD, B. J. Proactive inhibition in short-term retention of single items. Journal of Verbal Learning \& Verbal Behavior, 1962, 1, 153-161.

PETERSON, L. R., \& PETERSON, M. J. Short-term retention of individual verbal items. Journal of Experimental Psychology, 1959, 58, 193-198
THORNDIKE, E. L., \& LORGE, I The teacher's word book of 30,000 words. New York Columbia University Press, 1944.

\section{E. H. MURPHY ${ }^{2}$ and P. H. VENABLE $\bar{E}$, Birkbeck College, London, England}

The performance of the right and left ears in detection of two clicks does not differ significantly when clicks are presented alone. However, presentation of a brief shock $100 \mathrm{msec}$ before click presentation results in a considerable decrement in right-ear performance, but little change in left-ear performance. Results are discussed in relation to functional asymmetry of the right and left cerebral hemispheres.

Neurophysiological studies of the auditory pathways in animals suggest that the crossed pathways give a larger cortical response to monaural stimulation than do the uncrossed pathways, and that binaural stimulation accentuates the relative dominance of the crossed pathways (Rosenzweig, 1951). On the assumption that a similar arrangement of the auditory pathways obtains in man, techniques of dichotic stimulation have been used to compare recall or recognition of stimuli presented to the right and left ears and hence indirectly to investigate differences in function between the right and left cerebral hemispheres. With right-handed Ss, when digits are presented dichotically, recall is superior for digits presented to the right ear, and when melodies are presented dichotically, recognition is superior for melodies presented to the left ear. This finding has been interpreted as reflecting the predominant role of the left hemisphere in perception of verbal material and the predominant role of the right hemisphere in perception of nonverbal material (Kimura, $1961,1964)$. Studies of patients with unilateral temporal lobectomy have supported this interpretation (Milner, 1962). However, other investigators have
WICKENS, D. D. Encoding categories in short-term memory. Division 3 Presidential Address, American Psychological Association, 1968.

WICKENS, D. D., BORN, D. G., \& ALLEN, C. K. Proactive inhibition and item similarity in short-term memory. Journal of Verbal Learning \& Verbal Behavior, 1963, 2, 440-445. NOTES

1. This research was supported by the Advanced Research Projects Agency, Department of Defense, and was monitored by the Air Force Office of Scientific Research, under Contract No. AF 49(638)-1736 with Human Performance Center, Department of Psychology.

2. Now at the University of Minnesota, Minneapolis, Minn.

\section{Effects of ipsilateral and contralateral shock on ear asymmetry in the detection of two clicks ${ }^{1}$}

suggested that the ear asymmetry effects reported may be a function of attention bias (Treisman \& Geffen, 1968) or memory processes (Oxbury, Oxbury, \& Gardiner, 1967).

In a preliminary study, it was found that pairs of clicks presented to the left ear were better resolved than were pairs of clicks presented to the right ear. Presentation of a burst of white noise to the ear contralateral to that receiving the clicks had little effect on left-ear performance but caused a considerable decrement in rightear performance, thus accentuating the left-ear superiority. This finding accords with the neurophysiological evidence cited (Rosenzweig, 1951). However, it is possible that the results obtained were not unique to physiological mechanisms of the auditory pathways, but reflected hemispheric differences that were not modality specific. To examine this possibility, an experiment was carried out to investigate the effect of prior shock stimulation on right/left ear differences in the detection of two clicks.

\section{METHOD}

Clicks were delivered monaurally through Telephonics Type TDH.39 earphones, energized by square pulses, $0.1 \mathrm{msec}$ in duration, and triggered by a sequence timer that controlled the interval between the pulses. Clicks were approximately $90 \mathrm{~dB}$ in intensity. Shock was delivered through a pair of silver, rubber-bound electrodes, used with electrode jelly containing a saturated solution of $\mathrm{NaCl}$, placed approximately 2 in. apart on the ventral surface of the forearm and attached with adhesive tape. The shock consisted of a square pulse, $1 \mathrm{msec}$ in duration, triggered by the same timer as the clicks. An absolute threshold for sensation of shock was obtained for each arm; this level was then increased by $10 \mathrm{~V}$, and the level of each arm was adjusted slightly until 